\title{
HILBERT SPACE COMPRESSION UNDER DIRECT LIMITS AND CERTAIN GROUP EXTENSIONS
}

\author{
DENNIS DREESEN
}

(Communicated by Alexander N. Dranishnikov)

\begin{abstract}
We find bounds on the Hilbert space compression exponent of the limit of a directed metric system of groups. We also give estimates on the Hilbert space compression exponent of a group extension of a group $H$ by a word-hyperbolic group or a group of polynomial growth.
\end{abstract}

\section{INTRODUCTION}

In [6], Gromov introduced the notion of uniform embeddability of a finitely generated group into a Hilbert space and he believed that such groups would satisfy the Novikov conjecture [7. Six years later, Yu showed that such groups satisfy the coarse Baum-Connes conjecture [17, which is related to the Novikov conjecture. Together with Skandalis and $\mathrm{Tu}$, the Novikov conjecture for uniformly embeddable groups was eventually proven in [14].

Definition 1.1. A metric space $(X, d)$ is uniformly embeddable in a Hilbert space if there exist a Hilbert space $\mathcal{H}$, non-decreasing functions $\rho_{-}, \rho_{+}: \mathbb{R}^{+} \rightarrow \mathbb{R}^{+}$such that $\lim _{t \rightarrow \infty} \rho_{-}(t)=+\infty$, and a map $f: X \rightarrow \mathcal{H}$, such that

$$
\rho_{-}(d(x, y)) \leq\|f(x)-f(y)\| \leq \rho_{+}(d(x, y)) \forall x, y \in X .
$$

The map $f$ is called a uniform embedding of $X$ in $\mathcal{H}$. It is called large-scale Lipschitz whenever $\rho_{+}$can be taken to be of the form $\rho_{+}: t \mapsto C t+D$ for some $C>0, D \geq 0$. It is Lipschitz if we can take $D=0$.

It appears interesting to quantify the degree to which a metric space embeds uniformly into a Hilbert space [8]. This leads to the definition of the Hilbert space compression exponent.

Definition 1.2. Let $(X, d)$ be a metric space. Assume that $f:(X, d) \rightarrow \mathcal{H}$ is a uniform embedding of $X$ into a Hilbert space $\mathcal{H}$. The supremum of $r \in[0,1]$ such that there exist $C \geq 1, D \geq 0$ such that

$$
\forall x, y \in X: \frac{1}{C} d(x, y)^{r}-D \leq\|f(x)-f(y)\| \leq C d(x, y)+D
$$

is called the compression exponent of $f$ and is denoted by $R(f)$. The Hilbert space compression exponent (or Hilbert space compression) $\alpha(X)$ of $(X, d)$ is defined as

Received by the editors March 18, 2011 and, in revised form, June 16, 2011 and June 30, 2011. 2010 Mathematics Subject Classification. Primary 20F65; Secondary 20F67.

Key words and phrases. Hilbert space compression, hyperbolic groups, groups of polynomial growth, direct limits of groups.

The author is a research assistant for the Research Foundation - Flanders. 
the supremum of $R(f)$ over all large-scale Lipschitz uniform embeddings of $X$ into Hilbert spaces.

Remark 1.3. In the above definition, one considers only large-scale Lipschitz uniform embeddings. This is a natural convention as every uniform embedding of a quasi-geodesic metric space into a Hilbert space is large-scale Lipschitz (Proposition 2.9 in [8]). This holds in particular for the case that $X$ is a finitely generated group and $d$ is the word length metric relative to a finite symmetric generating subset.

The value of the Hilbert space compression exponent is related to other mathematical properties. In [8] for example, it is shown that a finitely generated group $G$, equipped with the word length metric relative to a finite symmetric generating subset, has Yu's property $(A)$ whenever $\alpha(G)>1 / 2$.

In our proofs, we make use of the following equivalent definition of uniform embeddability [3].

Definition 1.4. Let $(X, d)$ be a metric space. Then $X$ is uniformly embeddable in a Hilbert space if and only if for every $R, \epsilon>0$ there exist a Hilbert space valued map $\xi: X \rightarrow \mathcal{H}, x \rightarrow \xi^{x}$ such that $\left\|\xi^{x}\right\|=1$ for all $x \in X$ and such that

(1) $\left\|\xi^{x}-\xi^{x^{\prime}}\right\| \leq \epsilon$ provided $d\left(x, x^{\prime}\right) \leq R$,

(2) $\lim \sup _{S \rightarrow \infty}\left\{\left|\left\langle\xi^{x}, \xi^{y}\right\rangle\right|, d(x, y) \geq S\right\}=0$.

Knowing that Hilbert space compression quantifies uniform embeddability, we describe in this paper where exactly that the Hilbert space compression exponent is hiding in the above definition. This is used to prove Theorem 3.2 , where given a sequence

$$
G_{1} \stackrel{i_{1}}{\hookrightarrow} G_{2} \stackrel{i_{2}}{\hookrightarrow} \ldots
$$

of groups $G_{j}$ with length functions such that every $i_{j}$ is an isometric group homomorphism, we relate the compression exponents of the $G_{i}$ to that of their direct limit $G$.

After elaborating on a way to quantify Yu's property $(A)$, we will consequently be able to investigate how the Hilbert space compression exponent behaves under extensions by hyperbolic groups or groups of polynomial growth, both examples of groups with property $(A)$. Here, we also make use of methods from [3]. In the case of finitely generated groups, we obtain the following result in Section 4 (see Theorems 4.5 and 4.8).

Theorem 1.5. Assume that $\Gamma$ is a finitely generated group, equipped with the word length function $l$ relative to a finite symmetric generating subset $S$, that fits in a short exact sequence

$$
1 \rightarrow H \rightarrow \Gamma \stackrel{\pi}{\rightarrow} G \rightarrow 1
$$

Equip $H$ with the induced metric from $\Gamma$, denote the compression exponent of $H$ relative to this length function by $\delta$ and equip $G$ with the word length $l_{G}$ relative to $\pi(S)$.

(1) If $\left(G, l_{G}\right)$ has polynomial growth, then the compression exponent of $\Gamma$ is at least $\delta / 3$.

(2) If $\left(G, l_{G}\right)$ is hyperbolic, then the compression exponent of $\Gamma$ is at least $\delta / 5$. 


\section{A different way to interpret the Hilbert space COMPRESSION EXPONENT}

In this section, we explain where the Hilbert space compression exponent is hiding in Definition 1.4 of the Introduction. For the reader's convenience, we give the proof by Guentner and Dadarlat which shows the equivalence between Definitions 1.1 and 1.4

Proposition 2.1 (Proposition 2.1 in [3]). Let $(X, d)$ be a metric space. Then $X$ is uniformly embeddable in a Hilbert space as in Definition 1.1 if and only if for every $R>0$ and $\epsilon>0$ there exists a Hilbert space valued map $\xi: X \rightarrow \mathcal{H}, x \rightarrow \xi_{x}$ such that $\left\|\xi_{x}\right\|=1$ for all $x \in X$ and such that

(1) $\sup \left\{\left\|\xi_{x}-\xi_{x^{\prime}}\right\|: d\left(x, x^{\prime}\right) \leq R, x, x^{\prime} \in X\right\} \leq \epsilon$,

(2) $\liminf _{S \rightarrow \infty}\left\{\left\|\xi_{x}-\xi_{x^{\prime}}\right\|: d\left(x, x^{\prime}\right) \geq S, x, x^{\prime} \in X\right\}=\sqrt{2}$.

The second condition can be replaced by

$$
\limsup _{S \rightarrow \infty}\left\{\left|\left\langle\xi_{x}, \xi_{x^{\prime}}\right\rangle\right|: d\left(x, x^{\prime}\right) \geq S, x, x^{\prime} \in X\right\}=0 .
$$

Proof. Assume that $X$ is uniformly embeddable as in Definition 1.1 and let $F$ : $X \rightarrow \mathcal{H}$ be a uniform embedding of $X$ in a real Hilbert space $\mathcal{H}$. Let $\rho_{-}$and $\rho_{+}$ be functions such that

$$
\rho_{-}(d(x, y)) \leq\|F(x)-F(y)\| \leq \rho_{+}(d(x, y)) .
$$

Denote

$$
\operatorname{Exp}(\mathcal{H})=\mathbb{R} \oplus \mathcal{H} \oplus(\mathcal{H} \otimes \mathcal{H}) \oplus(\mathcal{H} \otimes \mathcal{H} \otimes \mathcal{H}) \oplus \cdots
$$

and define $\operatorname{Exp}: \mathcal{H} \rightarrow \operatorname{Exp}(\mathcal{H})$ by

$$
\operatorname{Exp}(\zeta)=1 \oplus \zeta \oplus\left(\frac{1}{\sqrt{2 !}} \zeta \otimes \zeta\right) \oplus\left(\frac{1}{\sqrt{3 !}} \zeta \otimes \zeta \otimes \zeta\right) \oplus \cdots
$$

Note that $\left\langle\operatorname{Exp}(\zeta), \operatorname{Exp}\left(\zeta^{\prime}\right)\right\rangle=e^{\left\langle\zeta, \zeta^{\prime}\right\rangle}$, for all $\zeta, \zeta^{\prime} \in \mathcal{H}$. For $t>0$ define

$$
\xi_{x}=e^{-t\|F(x)\|^{2}} \operatorname{Exp}(\sqrt{2 t} F(x)) .
$$

It is easily verified that $\left\langle\xi_{x}, \xi_{x^{\prime}}\right\rangle=e^{-t\left\|F(x)-F\left(x^{\prime}\right)\right\|^{2}}$. Consequently, for all $x, x^{\prime} \in X$ we have $\left\|\xi_{x}\right\|=1$, and

$$
e^{-t \rho_{+}\left(d\left(x, x^{\prime}\right)\right)^{2}} \leq\left\langle\xi_{x}, \xi_{x^{\prime}}\right\rangle \leq e^{-t \rho_{-}\left(d\left(x, x^{\prime}\right)\right)^{2}} .
$$

Putting $t=\frac{-\ln \left(1-\epsilon^{2} / 2\right)}{\rho_{+}(R)^{2}}$, it is easy to verify conditions (1) and (2) above.

Conversely, choose $p>0$ and assume that $X$ satisfies the conditions in the statement. There exist a sequence of maps $\eta_{n}: X \rightarrow \mathcal{H}_{n}$ and a sequence of numbers $S_{0}=0<S_{1}<S_{2}<\ldots$, increasing to infinity, such that for every $n \geq 1$ and every $x, x^{\prime} \in X$,

(1) $\left\|\eta_{n}(x)\right\|=1$;

(2) $\left\|\eta_{n}(x)-\eta_{n}\left(x^{\prime}\right)\right\| \leq \frac{1}{n^{1 / 2+p}}$, provided $d\left(x, x^{\prime}\right) \leq \sqrt{n}$ (actually, Guentner and Dadarlat use $\frac{1}{n}$ instead of $\frac{1}{n^{1 / 2+p}}$, but the latter bound is better for our purposes);

(3) $\left\|\eta_{n}(x)-\eta_{n}\left(x^{\prime}\right)\right\| \geq 1$, provided $d\left(x, x^{\prime}\right) \geq S_{n}$. 
Choose a base point $x_{0} \in X$ and define $F: X \rightarrow \bigoplus_{n=1}^{\infty} \mathcal{H}_{n}$ by

$$
F(x)=\frac{1}{2}\left(\left(\eta_{1}(x)-\eta_{1}\left(x_{0}\right)\right) \oplus\left(\eta_{2}(x)-\eta_{2}\left(x_{0}\right)\right) \oplus \cdots\right) .
$$

It is not hard to verify that $F$ is well defined and

$$
\rho_{-}\left(d\left(x, x^{\prime}\right)\right) \leq\left\|F(x)-F\left(x^{\prime}\right)\right\| \leq d\left(x, x^{\prime}\right)+C \text {, for all } x, x^{\prime} \in X,
$$

where $C>0$ is some constant, $\rho_{-}=\frac{1}{2} \sum_{n=1}^{\infty} \sqrt{n-1} \chi_{\left[S_{n-1}, S_{n}\right)}$, and the $\chi_{\left[S_{n-1}, S_{n}\right)}$ are the characteristic functions of the sets $\left[S_{n-1}, S_{n}\right)$.

Indeed, let $x, x^{\prime} \in X$. If $n$ is such that $\sqrt{n-1} \leq d\left(x, x^{\prime}\right)<\sqrt{n}$, we have

$$
\begin{aligned}
\left\|F(x)-F\left(x^{\prime}\right)\right\|^{2} & =\frac{1}{4} \sum_{i \leq n-1}\left\|\eta_{i}(x)-\eta_{i}\left(x^{\prime}\right)\right\|^{2}+\frac{1}{4} \sum_{i \geq n}\left\|\eta_{i}(x)-\eta_{i}\left(x^{\prime}\right)\right\|^{2} \\
& \leq(n-1)+\frac{1}{4} \sum_{i \geq n} \frac{1}{i^{1+2 p}} \leq d\left(x, x^{\prime}\right)^{2}+C,
\end{aligned}
$$

where $C=\frac{1}{4} \sum_{i \geq n} \frac{1}{i^{1+2 p}}<\infty$.

Similarly, if $n$ is such that $S_{n-1} \leq d\left(x, x^{\prime}\right)<S_{n}$, we have

$$
\left\|F(x)-F\left(x^{\prime}\right)\right\|^{2} \geq \frac{1}{4} \sum_{i \leq n-1}\left\|\eta_{i}(x)-\eta_{i}\left(x^{\prime}\right)\right\|^{2} \geq \frac{n-1}{4}=\rho_{-}\left(d\left(x, x^{\prime}\right)\right)^{2} .
$$

The following lemmas are crucial in what follows.

Lemma 2.2. Assume that $(X, d)$ is a metric space with compression exponent $\alpha>0$. Fix numbers $a, b, r \in \mathbb{R}^{+}$and set $R_{n}=n^{r}, \epsilon_{n}=\frac{1}{a n^{b}}$ for all $n \in \mathbb{N}_{0}$. For any $0<p<\alpha$ and every $n$ larger than some number $M_{p}$, we can find a collection of unit vectors $\left(\xi_{n}^{x}\right)_{x \in X}$ in some Hilbert space $\mathcal{H}_{p}$ satisfying

(1) $\left\|\xi_{n}^{x}-\xi_{n}^{y}\right\| \leq \epsilon_{n}$ provided $d(x, y) \leq R_{n}$,

(2) $\left\|\xi_{n}^{x}-\xi_{n}^{y}\right\| \geq 1$ provided $d(x, y) \geq S_{n}:=n^{\frac{r+b+p}{\alpha-p}}$.

The second condition can be replaced by

$$
\left|\left\langle\xi_{n}^{x}, \xi_{n}^{y}\right\rangle\right| \leq \frac{1}{2} \text { provided } d(x, y) \geq S_{n}:=n^{\frac{r+b+p}{\alpha-p}} .
$$

Proof. Let $F: X \rightarrow \mathcal{H}$ be a uniform embedding of $X$ into a Hilbert space satisfying

$$
\forall x, y \in X: \frac{1}{C} d(x, y)^{\alpha-p}-D \leq\|F(x)-F(y)\| \leq \widetilde{C} d(x, y)+\widetilde{D},
$$

for some $C, \widetilde{C}>0$ and $D, \widetilde{D} \geq 0$. Denote $\rho_{-}(d(x, y)):=\frac{1}{C} d(x, y)^{\alpha-p}-D$ and $\rho_{+}(d(x, y)):=\widetilde{C} d(x, y)+\widetilde{D}$. Fix $n \in \mathbb{N}_{0}$ and in the proof of Proposition 2.1, set $t=t_{n}=\frac{-\ln \left(1-\frac{\epsilon_{n}^{2}}{2}\right)}{\rho_{+}\left(R_{n}\right)^{2}}$ to obtain vectors $\left(\xi_{n}^{x}\right)_{x \in X}$ such that

$$
e^{-t_{n} \rho_{+}\left(d\left(x, x^{\prime}\right)\right)^{2}} \leq\left\langle\xi_{n}^{x}, \xi_{n}^{x^{\prime}}\right\rangle \leq e^{-t_{n} \rho_{-}\left(d\left(x, x^{\prime}\right)\right)^{2}} .
$$

It is easy to verify that the vectors $\left(\xi_{n}^{x}\right)_{x \in X}$ satisfy condition (1) of this corollary. Regarding the second condition, note that

$$
\begin{aligned}
\left\|\xi_{n}^{x}-\xi_{n}^{y}\right\|^{2} & =2-2\left\langle\xi_{n}^{x}, \xi_{n}^{y}\right\rangle \\
& \geq 2-2 e^{-t_{n} \rho_{-}(d(x, y))^{2}} \\
& =2-2 e^{\frac{\ln \left(1-\frac{\epsilon_{n}^{2}}{2}\right)}{\left(\widetilde{C} R_{n}+\bar{D}\right)^{2}}\left((1 / C) d(x, y)^{\alpha-p}-D\right)^{2}} .
\end{aligned}
$$


Consequently we have $\left\|\xi_{n}^{x}-\xi_{n}^{y}\right\| \geq 1$ whenever

$$
2-2 e^{\frac{\ln \left(1-\frac{\epsilon_{n}^{2}}{2}\right)}{\left(\tilde{C} R_{n}+\bar{D}\right)^{2}}\left((1 / C) d(x, y)^{\alpha-p}-D\right)^{2}} \geq 1,
$$

i.e. whenever

$$
\left(1-\frac{\epsilon_{n}^{2}}{2}\right)^{\frac{\left((1 / C) d(x, y)^{\alpha-p}-D\right)^{2}}{\left(\widetilde{C} R_{n}+\widetilde{D}\right)^{2}}} \leq \frac{1}{2} .
$$

This is true if and only if

$$
\frac{(1 / C) d(x, y)^{\alpha-p}-D}{\widetilde{C} R_{n}+\widetilde{D}} \geq \sqrt{\frac{-\ln (2)}{\ln \left(1-\frac{\epsilon_{n}^{2}}{2}\right)}}
$$

if and only if

$$
d(x, y) \geq\left[C\left(\sqrt{\frac{-\ln (2)}{\ln \left(1-\frac{1}{2 a^{2} n^{2 b}}\right)}}\left(\widetilde{C} n^{r}+\widetilde{D}\right)+D\right)\right]^{\frac{1}{\alpha-p}} .
$$

Since $\frac{-\ln (2)}{\ln \left(1-\frac{1}{2 a^{2} n^{2 b}}\right)} \leq \ln (2) 2 a^{2} n^{2 b} \leq 2 a^{2} n^{2 b}$, it suffices to take

$$
d(x, y) \geq\left[C \sqrt{2} a n^{b}\left(\widetilde{C} n^{r}+\widetilde{D}\right)+C D\right]^{\frac{1}{\alpha-p}}:=A_{n} .
$$

If $n$ is large enough, then $A_{n} \leq n^{\frac{b+r+p}{\alpha-p}}$, so we obtain $\left\|\xi_{n}^{x}-\xi_{n}^{y}\right\| \geq 1$ provided $d(x, y) \geq n^{\frac{r+b+p}{\alpha-p}}$.

This lemma indicates that the compression exponent hides in the speed that $\left(S_{n}\right)_{n}$ tends to infinity. The following lemma serves as some kind of converse.

Lemma 2.3. Let $(X, d)$ be a metric space. If for some $a, p>0$ and for every $n \in \mathbb{N}_{0}$ greater than some natural number $M$, one can find a collection of unit vectors $\left(\xi_{n}^{x}\right)_{x \in X}$ in a Hilbert space $\mathcal{H}_{n}$ such that

(1) $\left\|\xi_{n}^{x}-\xi_{n}^{y}\right\| \leq \frac{1}{n^{1 / 2+p}}$ provided $d(x, y) \leq \sqrt{n}$,

(2) $\left|\left\langle\xi_{n}^{x}, \xi_{n}^{y}\right\rangle\right| \leq \frac{1}{2}$ provided $d(x, y) \geq n^{a}$,

then the compression exponent of $X$ is greater than $\frac{1}{2 a}$.

Proof. It follows directly from the second part of the proof of Proposition 2.1 that the map

$$
\begin{aligned}
F: X & \rightarrow \bigoplus_{n \geq M} \mathcal{H}_{n} \\
x & \mapsto \frac{1}{2} \bigoplus_{n \geq M}\left(\xi_{n}^{x}-\xi_{n}^{x_{0}}\right)
\end{aligned}
$$

is a uniform embedding. The first condition on the vectors $\xi_{n}^{x}$ shows that $F$ is large-scale Lipschitz. From the second condition, we get that

$$
\forall x, y \in X:\|F(x)-F(y)\| \geq \frac{1}{2} \sum_{n=0}^{\infty} \sqrt{n+1} \chi_{\left[(M+n)^{a},(M+n+1)^{a}\right)}(d(x, y)) .
$$

This implies that the compression exponent of $F$ is at least $\beta$ if

$$
(M+n+1)^{a \beta} \leq \frac{\sqrt{n+1}}{2},
$$

for all $n$ sufficiently large. One derives easily that the compression exponent of $(X, d)$ is at least $\frac{1}{2 a}$. 
Recall that a metric space $(X, d)$ is called quasi-geodesic if there exist some $\delta, \lambda>0$ such that for every $x, y \in X$, there exists some discrete subset $\{x=$ $\left.x_{0}, x_{1}, \ldots, x_{n}=y\right\} \subset X$ such that $d\left(x_{i}, x_{i+1}\right)<\delta$ for every $i \in\{0,1, \ldots, n-1\}$ and such that

$$
\lambda \sum_{i=0}^{n-1} d\left(x_{i}, x_{i+1}\right) \leq d(x, y) \leq \sum_{i=0}^{n-1} d\left(x_{i}, x_{i+1}\right) .
$$

Note that the upper bound is just a consequence of the triangle inequality. For reference's sake, we write the following.

Corollary 2.4. If in addition to the assumptions of Lemma 2.3. $(X, d)$ is a quasigeodesic metric space, then the first condition on the vectors $\xi_{n}^{x}$ in the formulation of Lemma 2.3 can be replaced by

$$
\left\|\xi_{n}^{x}-\xi_{n}^{y}\right\| \leq \frac{1}{n^{1 / 2+p}}, \text { provided } d(x, y) \leq \ln (n) .
$$

Proof. If $(X, d)$ is quasi-geodesic, then every uniform embedding is large-scale Lipschitz (Remark 1.3). The first condition in the formulation of Lemma 2.3 is only used to prove that the constructed uniform embedding $F$ is large-scale Lipschitz; hence we can relax this condition in the setting of quasi-geodesic spaces.

\section{The Hilbert SPACE COMPRESSION EXPONENT FOR THE LIMIT OF A DIRECTED SYSTEM OF GROUPS}

\subsection{Formulation of the result.}

Definition 3.1. A length function $l$ on a group $G$ is a function $l: G \rightarrow \mathbb{R}^{+}$ satisfying

(1) $l(x)=0 \Leftrightarrow x=1$,

(2) $\forall x \in G, l(x)=l\left(x^{-1}\right)$,

(3) $\forall x, y \in G, l(x y) \leq l(x)+l(y)$.

We say that $l$ is proper whenever

$$
\forall M \in \mathbb{R}^{+}:|\{x \in G \mid l(x) \leq M\}|<\infty .
$$

Every length function on $G$ induces a left-invariant metric on $G$ by $d(x, y)=$ $l\left(x^{-1} y\right), \forall x, y \in G$.

Let $G_{1} \hookrightarrow G_{2} \hookrightarrow G_{3} \hookrightarrow \ldots$ be a directed system of groups $G_{i}$ with length functions $l_{i}$ such that the maps $G_{i} \hookrightarrow G_{i+1}$ are isometric group homomorphisms. Denote by $G$ the direct limit of this system. By definition, $G$ can be seen as the disjoint union of all the $G_{i}$ divided by some equivalence relation. Define the induced length function $l$ on $G$ by $l(x):=\lim _{i} l_{i}(x)$. We proceed under the assumption that $l$ is a proper length function on $G$. In this section, we investigate how the Hilbert space compression exponent of $G$ is related to the Hilbert space compression exponents of the $G_{i}$.

To start, notice that every $G_{i}$ can be seen as a metric subspace of $G$ and so $\alpha(G) \leq \inf _{i \in \mathbb{N}} \alpha\left(G_{i}\right)$. There are cases in which equality holds, but this is certainly not always the case. Indeed, one can equip the group

$$
\mathbb{Z}^{(\mathbb{Z})}=\{f: \mathbb{Z} \rightarrow \mathbb{Z} \text { with finite support }\}=\{(f, a) \in \mathbb{Z} \imath \mathbb{Z} \mid a=0\}
$$


with the induced metric from $\mathbb{Z} \imath \mathbb{Z}$. This group is the direct limit of the family of subgroups $G_{n}:=\mathbb{Z}^{2 n+1}=\{f:[-n, n] \rightarrow \mathbb{Z}\}$, where each $\mathbb{Z}^{2 n+1}$ is equipped with the subspace metric from $\mathbb{Z}^{(\mathbb{Z})}$. Since this metric is quasi-isometric to the standard word length metric on $\mathbb{Z}^{2 n+1}$, we obtain $\mathbb{Z}^{(\mathbb{Z})}$ as a limit of groups with compression exponent 1 . However, in [1], it is shown that the compression exponent of $\mathbb{Z}^{(\mathbb{Z})}$ is at most $3 / 4$. In [11], it was shown later that the compression exponent actually equals $\frac{3}{4}$.

The fact that $\mathbb{Z}$ and $\mathbb{Z}^{(\mathbb{Z})}$ have different compression exponents, yet are both limits of groups of compression 1, implies that we will need to include more information on how the groups $G_{i}$ are embedded in their respective Hilbert spaces in order to say something useful about the Hilbert space compression exponent of the limit. We propose the following.

Theorem 3.2. Assume that $G$ is the direct limit of a directed metric system $G_{1} \hookrightarrow$ $G_{2} \hookrightarrow G_{3} \hookrightarrow \ldots$ of groups such that the maps $G_{i} \hookrightarrow G_{i+1}$ are isometric group homomorphisms. Assume moreover that the induced length function $l$ on $G$ is proper.

If $\inf _{i \in \mathbb{N}}\left(\alpha\left(G_{i}\right)\right)=0$, then $\alpha(G)=0$.

Else, choose $0<\delta<\inf _{i \in \mathbb{N}} \alpha\left(G_{i}\right)$ and choose for every $i \in \mathbb{N}$, a Hilbert space $\mathcal{H}_{i}$, constants $C_{i}, \widetilde{D_{i}}>0, \widetilde{C_{i}}, D_{i} \geq 0$ and a map $f_{i}: G_{i} \rightarrow \mathcal{H}_{i}$ satisfying

$$
\left(1 / C_{i}\right) d(x, y)^{\delta}-D_{i} \leq\left\|f_{i}(x)-f_{i}(y)\right\| \leq \widetilde{C_{i}} d(x, y)+\widetilde{D_{i}} \forall x, y \in G_{i} .
$$

Choose $g: \mathbb{N} \rightarrow \mathbb{N}$ such that for all $x \in G$ we have $x \in G_{g(n)}$ whenever $l(x) \leq \sqrt{n}$. Then,

$$
\alpha(G) \geq \liminf _{n \rightarrow \infty} \frac{(\delta / 2) \ln (n-1)}{\ln \left(C_{g(n)} \sqrt{2 \ln (2) n}\left(\widetilde{C_{g(n)}} \sqrt{n}+\widetilde{D_{g(n)}}\right)+C_{g(n)} D_{g(n)}\right)} .
$$

3.2. Examples. An application of the above formula occurs in the case that $G$ is an infinite direct sum of finite groups $G=F_{0} \oplus F_{1} \oplus F_{2} \oplus \ldots$ with $F_{0}=\{1\}$. Here, it is natural to equip $G$ with the length function $l$ defined by $l(x)=\min \{n \in$ $\left.\mathbb{N} \mid x \in \bigoplus_{i=0}^{n} F_{i}\right\}$, for all $x \in G$. Clearly, $G$ is the limit of the directed system $\left(\bigoplus_{i=0}^{n} F_{i}\right)_{n}$. Recalling the fact that finite groups have Hilbert space compression equal to 1 , we can apply Theorem 3.2 where $f_{n}$ is the 0 -map and where $C_{n}=\widetilde{D_{n}}=$ $1, D_{n}=n, \widetilde{C_{n}}=0$. One obtains $\alpha(G)=1$.

Assume that $H$ and $G$ are finitely generated groups. Theorem 3.2 can also be used to bound the compression exponent of the groups $G^{(H)}=\{(f, 1) \in G \nmid H\}$, equipped with the induced metric from $G$ ? $H$. For example, our result implies that $\alpha\left(\mathbb{Z}^{(\mathbb{Z})}\right) \geq 2 / 5$. It must be mentioned that the so-obtained lower bound is weaker than the value $3 / 4$ obtained in [11].

3.3. Proof of the result. We will need the following lemma for the proof of Theorem 3.2

Lemma 3.3. The map

$$
G: p \mapsto \liminf _{n \rightarrow \infty} \frac{(\delta / 2) \ln (n-1)}{\ln \left(C_{g(n)}\left(\sqrt{2 \ln (2) n^{2 p+1}}\left(\widetilde{C_{g(n)}} \sqrt{n}+\widetilde{D_{g(n)}}\right)+D_{g(n)}\right)\right)}
$$

is continuous in $p=0$. 
Proof. Set $f(n)=(\delta / 2) \ln (n), h(n)=C_{g(n)} \sqrt{2 \ln (2) n}\left(\widetilde{C_{g(n)}} \sqrt{n}+\widetilde{D_{g(n)}}\right)$ and $u(n)=$ $C_{g(n)} D_{g(n)}$. Note that $G(p)=\liminf _{n \rightarrow \infty} \frac{f(n)}{\ln \left(h(n) n^{p}+u(n)\right)}$. For every $p \geq 0$, set

$$
E(p):=\liminf _{n \rightarrow \infty} \frac{f(n)}{\ln \left(n^{p}(h(n)+u(n))\right)}
$$

We have for all $p>0$ that

$$
E(p) \leq G(p) \leq E(0)=G(0)
$$

This implies that continuity of $E$ in $p=0$ implies continuity of $G$ in $p=0$. Now, to see why $E$ is continuous in $p=0$, let us first assume that there exists $p>0$ such that $E(p) \neq 0$. Then we can define $\tilde{E}=\exp (1 / E)$ in a neighbourhood of $\mathbb{R}^{+}$ around 0 . From

$$
\tilde{E}: p \mapsto \exp (1 / E(p))=\limsup _{n \rightarrow \infty}\left(n^{p}(h(n)+u(n))\right)^{\frac{2}{\delta \ln (n)}}=\exp (2 p / \delta) \tilde{E}(0),
$$

we deduce that $\tilde{E}$ and hence $E$ and $G$ are continuous in $p=0$.

Assume next that $E(p)=\frac{1}{2} \liminf _{n \rightarrow \infty} \frac{\delta \ln (n)}{p \ln (n)+\ln (h(n)+u(n))}=0$ for every $p>0$. From the fact that the term $p \ln (n)$ in the denominator has the same asymptotic behaviour as the numerator, we deduce that $E \equiv 0$ on a neighbourhood of 0 in $\mathbb{R}^{+}$.

Proof of the result. Choose $n \in \mathbb{N}_{0}, p>0$ and denote $R=\sqrt{n}, \epsilon=\frac{1}{n^{1 / 2+p}}$. Next, take $g(n) \in \mathbb{N}$ such that $x \in G_{g(n)}$ whenever $l(x) \leq R=\sqrt{n}$. Set $t=\frac{-\ln \left(1-\epsilon^{2} / 2\right)}{\left(\widetilde{\left.C_{g(n)} R+\widetilde{D_{g(n)}}\right)^{2}}\right.}$ and take vectors $\left(\xi_{x}\right)_{x \in G_{g(n)}}$ as in the proof of Proposition 2.1, i.e. such that for all $x, y \in G_{g(n)}:$

$$
e^{-t\left(\widetilde{C_{g(n)}} d_{g(n)}(x, y)+\widetilde{D_{g(n)}}\right)^{2}} \leq\left\langle\xi_{x}, \xi_{y}\right\rangle \leq e^{-t\left(\left(1 / C_{g(n)}\right) d_{g(n)}(x, y)^{\delta}-D_{g(n)}\right)^{2}} .
$$

From the lower bound on $\left\langle\xi_{x}, \xi_{y}\right\rangle$, one derives

$$
\left\|\xi_{x}-\xi_{y}\right\| \leq \epsilon \text { whenever } d_{g(n)}(x, y) \leq R \text {. }
$$

Calculating as in Lemma 2.2. we derive that $\left\|\xi_{x}-\xi_{y}\right\| \geq 1$ whenever $d_{g(n)}(x, y) \geq$ $S_{n}:=\left[C_{g(n)}\left(\sqrt{\frac{-\ln (2)}{\ln \left(1-\frac{1}{2 n^{2 p+1}}\right)}}\left(\widetilde{C_{g(n)}} \sqrt{n}+\widetilde{D_{g(n)}}\right)+D_{g(n)}\right)\right]^{\frac{1}{\delta}}$.

In the proof of Proposition 3.1 of [3, Dadarlat and Guentner explain how the family $\left(\xi_{x}\right)_{x \in G_{g(n)}}$ can be extended to a family of unit vectors $\left(\hat{\xi}_{x}\right)_{x \in G}$ in a larger Hilbert space, but still satisfying similar inequalities. More precisely, we obtain unit vectors $\left(\hat{\xi}_{x}\right)_{x \in G}$ in a Hilbert space satisfying:

(1) $\left\|\xi_{x}-\xi_{y}\right\| \leq \frac{1}{n^{1 / 2+p}}$ whenever $d(x, y) \leq \sqrt{n}$;

(2) $\left\|\xi_{x}-\xi_{y}\right\| \geq 1$ whenever $d(x, y) \geq S_{n}$. 
From the proof of Proposition 2.1, we derive the existence of a large-scale uniform embedding of $G$ into a Hilbert space whose compression function $\rho_{-}$is greater than $\frac{1}{2} \sum_{n=1}^{\infty} \sqrt{n-1} \chi_{\left[S_{n-1}, S_{n}\right)}(t)$. Choose some $\beta \in[0,1]$, and define $\gamma: \mathbb{R}^{+} \rightarrow \mathbb{R}^{+}, t \mapsto$ $t^{\beta}$. If $\gamma$ eventually lies under some multiple of $\rho_{-}$, then the compression exponent of $G$ is greater than $\beta$. We claim that this is true as long as

$$
\beta<\liminf _{n \rightarrow \infty}\left\{\frac{(\delta / 2) \ln (n-1)}{\ln \left(C_{g(n)}\left(\sqrt{2 \ln (2) n^{2 p+1}}\left(\widetilde{C_{g(n)}} \sqrt{n}+\widetilde{D_{g(n)}}\right)+D_{g(n)}\right)\right.}\right\} .
$$

Indeed, noting that $\lim _{n \rightarrow \infty}\left[\left(\frac{-\ln (2)}{\ln \left(1-\frac{1}{2 n^{2 p+1}}\right)}\right) /\left(2 \ln (2) n^{2 p+1}\right)\right]=1$, the above inequality is equivalent to

$$
\beta<\liminf _{n \rightarrow \infty}\left\{\frac{(\delta / 2) \ln (n-1)}{\ln \left(C_{g(n)}\left(\sqrt{\frac{-\ln (2)}{\ln \left(1-\frac{1}{2 n^{2 p+1}}\right)}}\left(\widetilde{C_{g(n)}} \sqrt{n}+\widetilde{D_{g(n)}}\right)+D_{g(n)}\right)\right)}\right\}
$$

If $\beta$ satisfies this equation, then

$$
\limsup _{n \rightarrow \infty} \frac{S_{n}^{\beta}}{\sqrt{n-1}}<\infty
$$

and so there is a constant $\bar{C}$ and some $T>0$ such that

$$
t^{\beta} \leq \bar{C} \rho_{-}(t), \forall t \geq T
$$

showing that $\alpha(G)>\beta$.

Finally now, recall that $p>0$ was arbitrarily chosen; i.e., the lower bound

$$
\liminf _{n \rightarrow \infty}\left\{\frac{(\delta / 2) \ln (n-1)}{\ln \left(C_{g(n)}\left(\sqrt{2 \ln (2) n^{2 p+1}}\left(\widetilde{C_{g(n)}} \sqrt{n}+\widetilde{D_{g(n)}}\right)+D_{g(n)}\right)\right)}\right.
$$

on the compression of $G$ holds for every $p$. In particular, by Lemma 3.3, we can let $p$ go to 0 and obtain the desired lower bound

$$
\liminf _{n \rightarrow \infty} \frac{(\delta / 2) \ln (n-1)}{\ln \left(C_{g(n)} \sqrt{2 \ln (2) n}\left(\widetilde{C_{g(n)}} \sqrt{n}+\widetilde{D_{g(n)}}\right)+C_{g(n)} D_{g(n)}\right)} .
$$

If $G$ happens to be a quasi-geodesic space, then we can use Corollary 2.4 to improve our result. Concretely, we can take $R=\ln (n)$ instead of $\sqrt{n}$ in the proof of Theorem 3.2 and so we obtain a smaller value for $S_{n}$. Using the same notation as in Theorem 3.2 and assuming that $G$ is a quasi-geodesic space, we obtain the following:

Denote by $g: \mathbb{N} \rightarrow \mathbb{N}$ a function such that for all $x \in G$ we have $x \in G_{g(n)}$ whenever $l(x) \leq \ln (n)$. Then,

$$
\alpha(G) \geq \limsup _{n \rightarrow \infty} \frac{(\delta / 2) \ln (n-1)}{\ln \left(C_{g(n)} \sqrt{2 \ln (2) n}\left(\widetilde{C_{g(n)}} \ln (n)+\widetilde{D_{g(n)}}\right)+C_{g(n)} D_{g(n)}\right)} .
$$

Remark 3.4. All of the above easily generalizes to directed systems of groups $\left(G_{i}\right)_{i \in I}$, where $I$ is any directed set. 


\section{The Behaviour of the Hilbert space Compression exponent UNDER GROUP EXTENSIONS}

In this section, we study the behaviour of the compression exponent under certain group extensions. With respect to this, we mention that there has been some previous work related to the behaviour of compression under a special type of group extension, namely wreath products. For example, S. Li showed that the wreath product of two groups with positive compression exponents again has positive compression exponent, but in his methods he does not really look at wreath products as extensions and his methods completely differ from ours [9]. Wreath products were also studied in [10, [15].

From now on, $\left(\Gamma, l_{\Gamma}\right)$ will denote a group that fits in a short exact sequence

$$
1 \rightarrow H \rightarrow \Gamma \stackrel{\pi}{\rightarrow} G \rightarrow 1
$$

On $H$, we consider the induced subspace metric. On $G$, it is natural to define the length of an $H$-coset $x H$ by $\inf \left\{l_{\Gamma}(y) \mid y \in x H\right\}$. In order for this to define a length function, we restrict ourselves to the case that $l_{\Gamma}$ is uniformly discrete.

We will elaborate on two cases: when $G$ is of polynomial growth and when $G$ is finitely generated hyperbolic. The idea behind the proof is to quantify the degree to which such a group satisfies Yu's property $(A)$.

Definition 4.1. A discrete group $(G, l)$ with $l$ proper is said to have Yu's property $(A)$ if for every $R>0$ and $\epsilon>0$ there exists an $S>0$ and a map $\xi: X \rightarrow l^{2}(G)$ such that for all $x, x^{\prime} \in X$ we have $\left\|\xi_{x}\right\|=1$ and

(1) $\left|1-\left\langle\xi_{x}, \xi_{x^{\prime}}\right\rangle\right| \leq \epsilon$ whenever $d\left(x, x^{\prime}\right) \leq R$,

(2) the support of $\xi_{x}$ lies inside $B_{S}(x)$ for all $x \in G$.

Clearly, property $(A)$ implies uniform embeddability.

\subsection{Extensions by a group of polynomial growth.}

Definition 4.2. A metric space $(X, d)$ has polynomial growth if there exists a polynomial $P$ such that $|\overline{B(x, R)}| \leq P(R)$ for every $x \in X$ and every $R \geq 0$. Here $\overline{B(x, R)}$ is the closed ball with radius $R$ and center $x$.

In [16, Tu proves that groups of polynomial growth have property A. We obtain the following lemma by quantifying his proof.

Lemma 4.3. Let $(G, l)$ be a group that has polynomial growth. Let $p \in] 0,1[$ be any real number. There exists $n_{0} \in \mathbb{N}$ such that for every natural number $n \geq n_{0}$, there exists a collection of unit vectors $\left(g_{n}(x)\right)_{x \in G}$ in $l^{2}(G)$ such that $\left\|g_{n}(x)\right\|_{2}=$ $1, \forall x \in G$ and

(1) $\left|1-\left\langle g_{n}(x), g_{n}(y)\right\rangle\right| \leq \frac{1}{4 n^{1+2 p}}$ provided $d_{G}(x, y) \leq \sqrt{n}$,

(2) $\operatorname{supp}\left(g_{n}(x)\right) \subset B\left(x, S_{n}^{G}\right)$ for all $x \in G$, where $S_{n}^{G}=n^{3 / 2+5 p}$.

Proof. For each $x \in G$ and $r \in \mathbb{R}$, denote by $B(x, r) \subset G$ the ball of radius $r$ and center $x$. Denote the characteristic function of $B(x, r)$ by $\chi_{x}^{r}$. We shall denote $B(1, r)$ simply by $B_{r}$ and $\chi_{1}^{r}$ by $\chi_{r}$. For $n \in \mathbb{N}_{0}$, denote $R_{n}=\sqrt{n}$ and, with the convention that $\forall a \in \mathbb{R}: a / 0=\infty$, let $\overline{m_{n}}$ be the infimum of all real numbers $r$ such that

$$
\frac{\left|B_{r+R_{n}}\right|}{\left|B_{r-R_{n}}\right|} \leq 1+\frac{1}{2 n^{1+2 p}} .
$$


Clearly, such an $\overline{m_{n}}$ exists, since if it did not exist, then $\forall i \in \mathbb{N}_{0}$,

$$
\left|B_{2 i R_{n}+R_{n}}\right| \geq\left|B_{2(i-1) R_{n}+R_{n}}\right|\left(1+\frac{1}{2 n^{1+2 p}}\right) \geq \ldots \geq\left|B_{R_{n}}\right|\left(1+\frac{1}{2 n^{1+2 p}}\right)^{i},
$$

obtaining a contradiction, since the left-hand side depends polynomially on $i$ whereas the right-hand side depends exponentially on $i$.

We claim that there exists $\bar{n} \in \mathbb{N}_{0}$ such that $\forall n \geq \bar{n}: \overline{m_{n}} \leq 2 n^{3 / 2+4 p}$. Assume therefore that such an $\bar{n}$ does not exist. Then there exists a strictly monotone increasing sequence $\left(n_{i}\right)_{i \in \mathbb{N}}$ such that

$$
\forall i: \frac{\left|B_{2 n_{i}^{3 / 2+4 p}+R_{n_{i}}}\right|}{\left|B_{2 n_{i}^{3 / 2+4 p}-R_{n_{i}}}\right|}>1+\frac{1}{2 n_{i}^{1+2 p}} .
$$

Denoting the integer part of a real number $a$ by $[a]$ and assuming for the last inequality below that $\forall i: n_{i}^{p} \geq 2$, we obtain that

$$
\begin{aligned}
\left|B_{2 n_{i}^{3 / 2+4 p}+R_{n_{i}}}\right| & >\left(1+\frac{1}{2 n_{i}^{1+2 p}}\right)\left|B_{2 n_{i}^{3 / 2+4 p}-2 R_{n_{i}}+R_{n_{i}}}\right| \\
& >\left(1+\frac{1}{2 n_{i}^{1+2 p}}\right)^{2}\left|B_{2 n_{i}^{3 / 2+4 p}}-4 R_{n_{i}}+R_{n_{i}}\right| \\
& >\ldots\left(1+\frac{1}{2 n_{i}^{1+2 p}}\right)^{\left[n_{i}^{1+4 p}\right]}\left|B_{R_{n_{i}}}\right| \\
& >\left(1+\frac{1}{2 n_{i}^{1+2 p}}\right)^{n_{i}^{1+3 p}}\left|B_{R_{n_{i}}}\right| .
\end{aligned}
$$

Since $\lim _{i \rightarrow \infty}\left(1+\frac{1}{2 n_{i}^{1+2 p}}\right)^{n_{i}^{1+2 p}}=\exp (1 / 2)$, it is clear that the right-hand side depends superpolynomially on $n_{i}$, whereas the left-hand side depends polynomially on $n_{i}$. We obtain a contradiction.

For $n \geq \bar{n}$, choose $m_{n}<2 n^{3 / 2+4 p} n^{p / 2}$ such that

$$
\frac{\left|B_{m_{n}+R_{n}}\right|}{\left|B_{m_{n}-R_{n}}\right|} \leq 1+\frac{1}{2 n^{1+2 p}} .
$$

Consider now the functions $\chi_{x}^{m_{n}}$. They are elements of $l^{1}(G)$ such that $d_{G}(x, y) \leq$ $\sqrt{n}=R_{n}$ implies that

$$
\begin{aligned}
\frac{\left\|\chi_{x}^{m_{n}}-\chi_{y}^{m_{n}}\right\|_{1}}{\left\|\chi_{x}^{m_{n}}\right\|_{1}} & \leq \frac{\left|B\left(x, m_{n}+R_{n}\right)\right|-\left|B\left(x, m_{n}-R_{n}\right)\right|}{\left|B\left(x, m_{n}-R_{n}\right)\right|} \\
& =\frac{\left|B_{m_{n}+R_{n}}\right|}{\left|B_{m_{n}-R_{n}}\right|}-1 \leq \frac{1}{2 n^{1+2 p}} .
\end{aligned}
$$

Moreover, the support of $\chi_{x}^{m_{n}}$ lies inside

$$
\overline{B\left(x, m_{n}\right)} \subset B\left(x, n^{3 / 2+5 p}\right)
$$

whenever $n$ is larger than some natural number $\overline{n_{1}}$. To conclude, take $n \geq n_{0}:=$ $\max \left(\bar{n}, \overline{n_{1}}\right)$ and define $g_{n}(x)=\sqrt{\frac{\chi_{x}^{m_{n}}}{\left\|\chi_{m_{n}}\right\|_{1}}}$. Clearly, these are elements of norm 1 in $l^{2}(G)$ that satisfy condition $(2)$ of this lemma. To show that they also satisfy 
condition (1), take $x, y$ such that $d_{G}(x, y) \leq R_{n}$. Then

$$
\begin{aligned}
\left\|g_{n}(x)-g_{n}(y)\right\|_{2}^{2} & =\sum_{z \in X}\left|g_{n}(x)(z)-g_{n}(y)(z)\right|^{2} \\
& \leq \sum_{z \in X}\left(\left|g_{n}(x)(z)-g_{n}(y)(z)\right| \cdot\left|g_{n}(x)(z)+g_{n}(y)(z)\right|\right) \\
& =\sum_{z \in X}\left|g_{n}(x)(z)^{2}-g_{n}(y)(z)^{2}\right| \\
& =\frac{\left\|\chi_{x}^{m_{n}}-\chi_{y}^{m_{n}}\right\|_{1}}{\left\|\chi_{m_{n}}\right\|_{1}} \leq \frac{1}{2 n^{1+2 p}}
\end{aligned}
$$

Therefore $\left|1-\left\langle g_{n}(x), g_{n}(y)\right\rangle\right| \leq \frac{1}{4 n^{1+2 p}}$ as desired.

Carefully modifying the proof above by setting $R_{n}:=\ln (n)$ and $m_{n}:=2 n^{1+4 p}$, we obtain the following.

Corollary 4.4. Let $G$ be a group that has polynomial growth. Let $p \in] 0,1[$ be any real number. There exists $n_{0} \in \mathbb{N}$ such that for every natural number $n \geq n_{0}$, there exists a collection of unit vectors $\left(g_{n}(x)\right)_{x \in G}$ in $l^{2}(G)$ such that $\left\|g_{n}(x)\right\|_{2}=1, \forall x \in$ $G$ and

(1) $\left|1-\left\langle g_{n}(x), g_{n}(y)\right\rangle\right| \leq \frac{1}{4 n^{1+2 p}}$ provided $d_{G}(x, y) \leq \ln (n)$,

(2) $\operatorname{supp}\left(g_{n}(x)\right) \subset B\left(x, S_{n}^{G}\right)$ for all $x \in G$, where $S_{n}^{G}=n^{1+5 p}$.

Theorem 4.5. Assume that $\Gamma$ is a group equipped with a uniformly discrete length function $l=l_{\Gamma}$ that fits in a short exact sequence

$$
1 \rightarrow H \rightarrow \Gamma \stackrel{\pi}{\rightarrow} G \rightarrow 1
$$

If $G$ with the induced metric from $\Gamma$ has polynomial growth and if the compression exponent of $H$ with the induced length $l_{H}:=l_{\mid \Gamma}$ equals $\delta$, then the compression exponent of $\Gamma$ is at least $\delta / 4$.

Proof. Denote the Hilbert space compression exponent of $H$ by $\delta>0$, choose $0<p<\delta$ and set $S_{n}^{G}$ as in Lemma 4.3. If $n$ is large enough, then $n^{3 / 2+6 p} \geq$ $2 S_{n}^{G}+\sqrt{n}=2 n^{3 / 2+5 p}+\sqrt{n}$. For $n$ sufficiently large, Lemma 2.2 applied for $r=3 / 2+6 p, a=\sqrt{2}, b=1 / 2+p$ gives a Hilbert space $\mathcal{H}$ and maps $h_{n}: H \rightarrow \mathcal{H}$ such that $\left\|h_{n}(s)\right\|=1 \forall s \in H$ and

- $\left|1-\left\langle h_{n}(s), h_{n}(\tilde{s})\right\rangle\right| \leq \frac{1}{4 n^{1+2 p}}$ provided $d_{H}(s, \tilde{s}) \leq 2 S_{n}^{G}+\sqrt{n}$,

- $\left|\left\langle h_{n}(s), h_{n}(\tilde{s})\right\rangle\right| \leq \frac{1}{2}$ whenever $d(s, \tilde{s}) \geq S_{n}^{H}:=n^{\frac{2+8 p}{\delta-p}}=\left(n^{1 / 2+3 p} S_{n}^{G}\right)^{\frac{1}{\delta-p}}$.

For $n$ sufficiently large, Lemma 4.3 provides maps $g_{n}: G \rightarrow l^{2}(G)$ such that $\left\|g_{n}(x)\right\|_{2}=1, \forall x \in G$ and such that

- $\left|1-\left\langle g_{n}(x), g_{n}(y)\right\rangle\right| \leq \frac{1}{4 n^{1+2 p}}$ provided $d_{G}(x, y) \leq \sqrt{n}$,

- $\operatorname{supp}\left(g_{n}(x)\right) \subset B\left(x, S_{n}^{G}\right)$ for all $x \in G$, where $S_{n}^{G}=n^{3 / 2+5 p}$.

In the proof of Theorem 4.1 in [3, Dadarlat and Guentner fix $n$ and use the maps $g_{n}$ and $h_{n}$ to construct a map $f_{n}: \Gamma \rightarrow l^{2}(G, \mathcal{H})$ such that $\left\|f_{n}(a)\right\|=1, \forall a \in \Gamma$ and

- $\left|1-\left\langle f_{n}(a), f_{n}(b)\right\rangle\right| \leq \frac{1}{2 n^{1+2 p}}$ if $d(a, b) \leq \sqrt{n}$,

- $\left\|f_{n}(a)-f_{n}(b)\right\| \geq 1$ if $d(a, b) \geq 2 S_{n}^{G}+S_{n}^{H}$. 
Denoting $\overline{S_{n}}=n^{p} S_{n}^{H}$, we obtain for $n$ larger than some $n_{1} \in \mathbb{N}_{0}$ that

- $\left\|f_{n}(a)-f_{n}(b)\right\| \leq \frac{1}{n^{1 / 2+p}}$ if $d(a, b) \leq \sqrt{n}$,

- $\left\|f_{n}(a)-f_{n}(b)\right\| \geq 1$ if $d(a, b) \geq \overline{S_{n}}$.

From Lemma 2.3, one then deduces that the compression exponent of $\Gamma$ is at least $\delta / 4$.

Remark 4.6. If $\left(\Gamma, l_{\Gamma}\right)$ is quasi-geodesic as a metric space, e.g. if it is a finitely generated group equipped with word length, then we can use Corollaries 2.4 and 4.4 to improve the lower bound $\delta / 4$ to $\delta / 3$.

4.2. Extensions by a finitely generated word-hyperbolic group. Let us start by a lemma similar to Lemma 4.3. Quantifying the proof of Proposition 8.1 in [16], we obtain

Lemma 4.7. Let $G$ be a finitely generated hyperbolic group (equipped with word length). Let $p \in] 0,1\left[\right.$ be any real number. There exists $n_{0} \in \mathbb{N}$ such that for every natural number $n \geq n_{0}$, there exists a collection of unit vectors $\left(g_{n}(x)\right)_{x \in G}$ in $l^{2}(G)$ such that $\left\|g_{n}(x)\right\|=1, \forall x \in G$ and

- $\left|1-\left\langle g_{n}(x), g_{n}(y)\right\rangle\right| \leq \frac{1}{4 n^{1+2 p}}$ provided $d_{G}(x, y) \leq \ln (n)$,

- $\operatorname{supp}\left(g_{n}(x)\right) \subset B\left(x, S_{n}^{G}\right)$ for all $x \in G$ where $S_{n}^{G}=n^{2+6 p}$.

Proof. Choose $a \in \partial G$, the Gromov boundary of $G$. For all $x \in G$, let [[x, $a[[$ be the set of infinite geodesics that belong to $a$ and such that $g(0)=x$. For every $x \in G$ and $k, n \in \mathbb{N}_{0}$, we define elements of $l^{1}(G)$ as follows:

$$
\begin{gathered}
F(x, k, n)=\text { characteristic function of } \bigcup_{\substack{d(x, y)<k \\
g \in[y, a[[}} g([n, 2 n]), \\
H(x, n)=\frac{1}{n^{3 / 2}} \sum_{k<\sqrt{n}} F(x, k, n) .
\end{gathered}
$$

It follows easily from these definitions that $\|F(x, k, n)\|_{1} \geq n$ for every $k, n \in \mathbb{N}_{0}$. Consequently, we have that $\|H(x, n)\|_{1} \geq 1$. Also, it is clear that the support of $H(x, n)$ is inside $B(x, \sqrt{n}+2 n)$. In the proof of Proposition 8.1 in [16, Tu shows that there is a constant $C>0$ such that for every $R>0$ :

$$
\|H(x, n)-H(y, n)\|_{l^{1}(G)} \leq \frac{2 C(R+1)}{n^{1 / 2}}, \text { whenever } d_{G}(x, y) \leq R .
$$

Let $m_{n}=n^{2+5 p}$ play the role of $n$. Then there exists $C>0$ such that the $H\left(x, m_{n}\right)_{x \in G, n \in \mathbb{N}_{0}}$ satisfy the following conditions for all $n$ greater than some natural number $n_{0}$ :

(1) $\left\|H\left(x, m_{n}\right)\right\|_{1} \geq 1$,

(2) $\left\|H\left(x, m_{n}\right)-H\left(y, m_{n}\right)\right\|_{1} \leq \frac{2 C(\ln (n)+1)}{n^{(2+5 p) 1 / 2}} \leq \frac{1}{2 n^{1+2 p}}$ provided $d_{G}(x, y) \leq \ln (n)$,

(3) $\operatorname{supp}\left(H\left(x, m_{n}\right)\right) \subset B\left(x, n^{2+6 p}\right)$,

where in (3), we use the fact that for $n$ sufficiently large: $2 m_{n}+\sqrt{m_{n}} \leq n^{2+6 p}$. For all $x \in G, n \geq n_{0}$, set $g_{n}(x)=\sqrt{\frac{H\left(x, m_{n}\right)}{\left\|H\left(1, m_{n}\right)\right\|_{1}}}$ to obtain a collection of elements of $l^{2}(G)$. Calculating as in the end of the proof of Lemma 4.3, we obtain

$$
\begin{aligned}
\left\|g_{n}(x)-g_{n}(y)\right\|_{2}^{2} & \leq \frac{\left\|H\left(x, m_{n}\right)-H\left(y, m_{n}\right)\right\|_{1}}{\left\|H\left(1, m_{n}\right)\right\|_{1}} \\
& \leq\left\|H\left(x, m_{n}\right)-H\left(y, m_{n}\right)\right\|_{1} .
\end{aligned}
$$


The $g_{n}(x)$ with $n \geq n_{0}$ satisfy the conditions of this lemma.

Theorem 4.8. Assume that $\Gamma$ is a finitely generated group, equipped with the word length function $l=l_{\Gamma}$ relative to some finite symmetric generating subset $S$ and that it fits in a short exact sequence

$$
1 \rightarrow H \rightarrow \Gamma \stackrel{\pi}{\rightarrow} G \rightarrow 1
$$

Equip $G$ with the word length function $l_{G}$ relative to $\pi(S)$. If $G$ is a finitely generated hyperbolic group in the sense of Gromov [5] and if $H$, with the induced metric from $\Gamma$, has Hilbert space compression exponent $\delta$, then the Hilbert space compression exponent of $\Gamma$ is at least $\delta / 5$.

Proof. The proof is analogous to the proof of Theorem 4.5. First, denoting $S_{n}^{G}=$ $n^{2+6 p}$ as in Lemma 4.7 and using Lemma 2.2 for $r=2+7 p, a=\sqrt{2}, b=(1 / 2)+p$, we find a Hilbert space $\mathcal{H}$ and unit vectors $\left(h_{n}(s)\right)_{s \in H} \in \mathcal{H}$ for every $n$ large enough such that

- $\left|1-\left\langle h_{n}(s), h_{n}(\tilde{s})\right\rangle\right| \leq \frac{1}{4 n^{1+2 p}}$ provided $d_{H}(s, \tilde{s}) \leq 2 S_{n}^{G}+\sqrt{n}$,

- $\left|\left\langle h_{n}(s), h_{n}(\tilde{s})\right\rangle\right| \leq \frac{1}{2}$ whenever $d(s, \tilde{s}) \geq S_{n}^{H}:=n^{\frac{(5 / 2)+9 p}{\delta-p}}=\left(n^{1 / 2+3 p} S_{n}^{G}\right)^{\frac{1}{\delta-p}}$.

Recall from Lemma 4.7 that, for $n$ large enough, we have proven the existence of maps $g_{n}: G \rightarrow l^{2}(G)$ such that $\left\|g_{n}(x)\right\|_{2}=1, \forall x \in G$ and such that

- $\left|1-\left\langle g_{n}(x), g_{n}(y)\right\rangle\right| \leq \frac{1}{4 n^{1+2 p}}$ provided $d_{G}(x, y) \leq \ln (n)$,

- $\operatorname{supp}\left(g_{n}(x)\right) \subset B\left(x, S_{n}^{G}\right)$ for all $x \in G$ where $S_{n}^{G}=n^{2+6 p}$.

The proof of Theorem 4.1 in 3 ] gives, for every $n \in \mathbb{N}$ larger than some $n_{1} \in \mathbb{N}$, a map $f_{n}: \Gamma \rightarrow l^{2}(G, \mathcal{H})$ such that $\left\|f_{n}(a)\right\|=1, \forall a \in \Gamma$ and

- $\left\|f_{n}(a)-f_{n}(b)\right\| \leq \frac{1}{n^{1 / 2+p}}$ if $d(a, b) \leq \ln (n)$,

- $\left\|f_{n}(a)-f_{n}(b)\right\| \geq 1$ if $d(a, b) \geq \overline{S_{n}}:=n^{p} S_{n}^{H}$.

Corollary 2.4 implies that the compression exponent of $\Gamma$ is at least $\delta / 5$.

Alain Valette pointed out that a stronger result is valid in the following special case.

Theorem 4.9. Let $A$ and $G$ be finitely generated groups, each equipped with the word length metric relative to a finite symmetric generating subset. Assume that $A$ is abelian, that $G$ is word-hyperbolic and that

$$
0 \rightarrow A \rightarrow \Gamma \stackrel{\pi}{\rightarrow} G \rightarrow 1
$$

is a central extension. The compression exponent of $\Gamma$, equipped with the word length metric relative to a finite symmetric generating subset, equals 1 .

Proof. Denote the second bounded cohomology group of $G$, defined using bounded cocycles, by $H_{b}^{2}(G, A)$. By [12], the comparison map

$$
H_{b}^{2}(G, A) \rightarrow H^{2}(G, A)
$$

is onto for $G$ word-hyperbolic; i.e., every 2-cocycle has a bounded representative.

Now, let $s: G \rightarrow \Gamma$ be a (set-theoretic) section, i.e. $\pi \circ s=I d_{G}$, and define

$$
c(x, y)=s(x y)^{-1} s(x) s(y) \forall x, y \in G .
$$


By the above, we can assume that $c$ is bounded and so Gersten's result [4], 13] implies that $\Gamma$ is quasi-isometric to $G \times A$. Consequently, the compression exponent of $\Gamma$ equals the minimum of the compression exponents of $G$ and $A$ [8], which is 1 .

\section{ACKNOWLEDGEMENTS}

The author thanks Alain Valette for reading previous versions of this article, for introducing him to the world of Hilbert space compression, for encouragement and for pointing out the result formulated in Theorem 4.9. He thanks Paul Igodt for encouragement, support and interesting conversations. He thanks Pieter Penninckx for useful suggestions and Nansen Petrosyan and Pierre de la Harpe for interesting conversations. Finally, he thanks the referee for useful suggestions and a crucial remark.

\section{REFERENCES}

[1] G.N. Arzhantseva, V.S. Guba, and M. V. Sapir, 'Metrics on diagram groups and uniform embeddings in a Hilbert space', Commentarii Mathematici Helvetici 81, No. 4 (2006), 911929. MR2271228 (2007k:20084)

[2] N. Brodskiy and D. Sonkin, 'Compression of uniform embeddings into Hilbert space', Topology and its Applications 155, No. 7 (2008), 725-732. MR2395586 (2009b:20078)

[3] M. Dadarlat and E. Guentner, 'Constructions preserving Hilbert space uniform embeddability of discrete groups', Transactions of the American Mathematical Society 355, no. 8 (2003) 3253-3275. MR 1974686 (2004e:20070)

[4] S.M. Gersten, 'Bounded cocycles and combings of groups', Internat. J. Algebra Comput. 2, no. 3 (1992), 307-326. MR 1189238 (93i:20029)

[5] E. Ghys and P. de la Harpe, 'Sur les groupes hyperboliques d'après Mikhael Gromov', Progress in Mathematics 83 (1990), Birkhäuser Boston. MR.1086648 (92f:53050)

[6] M. Gromov, Asymptotic invariants of infinite groups, Geometric Group Theory (A. Niblo and M. Roller, eds.), London Mathematical Society Lecture Notes 182 (1993), 1-295. MR.1253544 (95m:20041)

[7] M. Gromov, Problems (4) and (5), Novikov conjectures, index theorems and rigidity, Lecture Note Series, London Mathematical Society 226, Vol. 1 (1995) (S. Ferry, A. Ranicki and J. Rosenberg, editors). MR 1388294 (96m:57002)

[8] E. Guentner and J. Kaminker, 'Exactness and uniform embeddability of discrete groups', Journal of the London Mathematical Society 70, no. 3 (2004), 703-718. MR2160829 (2006i:43006)

[9] S. Li, 'Compression bounds for wreath products', Proceedings of the American Mathematical Society 138, no. 8 (2010), 2701-2714. MR2644886 (2011g:20061)

[10] A. Naor and Y. Peres, 'Embeddings of discrete groups and the speed of random walks', International Mathematics Research Notices 2008, Art. ID rnn 076. MR2439557 (2009m:20067)

[11] A. Naor and Y. Peres, ' $L_{p}$ compression, traveling salesmen, and stable walks', Duke Mathematical Journal 157, no. 1 (2011), 53-108. MR2783928

[12] W. D. Neumann and L. Reeves, 'Central extensions of word hyperbolic groups', Ann. of Math.(2) 145, no. 1 (1997), 183-192. MR1432040 (98b:20059)

[13] W. D. Neumann and L. Reeves, 'Regular cocycles and biautomatic structures', Internat. J. Algebra Comput. 6, no. 3 (1996), 313-324. MR1404809 (97h:20043)

[14] G. Skandalis, J. L. Tu, and G. Yu, Coarse Baum-Connes conjecture and groupoids, Topology 41 (2002), 807-834. MR1905840 (2003c:58020)

[15] R. Tessera, Asymptotic isoperimetry on groups and uniform embeddings into Banach spaces, Commentarii Mathematici Helvetici 86 (2011), 499-535. MR2803851

[16] J. Tu, 'Remarks on Yu's "property A" for discrete metric spaces and groups', Bull. Soc. Math. France 129, no. 1 (2001), 115-139. MR1871980(2002j:58038)

[17] G. Yu, The coarse Baum-Connes conjecture for spaces which admit a uniform embedding into Hilbert space, Invent. Math. 139, no. 1 (2001), 201-240. MR1728880 (2000j:19005) 
Katholieke Universiteit Leuven campus Kortrijk, Etienne Sabbelaan 53, 8500 Kortrijk, Belgium - And - Institut de Mathématiques, Université de Neuchâtel, Rue EmileArgand 11, 2009 Neuchâtel, Switzerland

E-mail address: dennis.dreesen@kuleuven-kortrijk.be; dennis.dreesen@unine.ch

Current address: Bât. M. de Hemptinne, á l'att. de Dennis Dreesen, Chemin du Cyclotron 2, 1348 Louvain-la-Neuve, Belgium

E-mail address: dennis.dreesen@uclouvain.be 